\title{
Confirmation of jailed side-branch ostium in coronary bifurcation intervention by stent-oriented three-dimensional intravascular ultrasound
}

\author{
Fumiaki Nakao \\ Department of Cardiology, Yamaguchi Grand Medical Center, Ohsaki, Hofu, Yamaguchi, Japan
}

The confirmation of jailing type and rewiring position on the side-branch ostium (SBO) by the three-dimensional (3D) optical coherence tomography (OCT)/optical frequency domain imaging (OFDI) is reported to lead to good stent apposition in percutaneous coronary intervention $(\mathrm{PCI})$ for bifurcation lesion [1-3].

The technology of intravascular ultrasound (IVUS) is advancing, AltaView and VISICUBE (a high-definition IVUS imaging catheter and console, TERUMO, Tokyo, Japan) has an ultrasound frequency of 40 or $60 \mathrm{MHz}$, a maximum frame rate of $90 \mathrm{frames} / \mathrm{s}$, and a maximum pullback speed (PBS) of $9 \mathrm{~mm} / \mathrm{s}$. Generally, a faster PBS reduces motion artifacts, but becomes a frame pitch wider. Because of its high frame rate, the narrowest frame pitch at maximum PBS of AltaView and VISICUBE is $0.1 \mathrm{~mm} /$ frame, which is the same as a frame pitch of OCT/OFDI that can make stent-enhanced 3D-images [1]. In this case a 3D-reconstructed IVUS image was made that could confirm jailing type and rewiring position on the jailed SBO.

A 2-link 8-crown bioresorbable polymer sirolimus-eluting stent (Ultimaster Tansei, TERUMO) was simply deployed on a coronary artery in the present clinical case. To segment the vessel lumen, a flushing of blood cells by contrast medium was undergone at image collecting by AltaView (a setting of $60 \mathrm{MHz}, 90 \mathrm{frames} / \mathrm{s}$, and PBS $9 \mathrm{~mm} / \mathrm{s}$ ). The IVUS image data was transferred to an offline Windows computer as an audio video interleaving (AVI) format using a universal serial bus (USB) flush drive. This image file was a compressed AVI format, converted to an uncompressed AVI format using the freeware AviUtl version 1.00 [4], and converted to a stacked tagged image file format (TIFF) using the freeware Image $1.43 \mathrm{v}$ [5]. Initially, the 3D-IVUS was simply reconstructed from the original IVUS images (stacked TIFF) (Fig. 1A). Confirmation of struts and links in the original 3D-IVUS was difficult because of the blooming effect of strut signals. ImageJ can process various filters for images. The minimum filter does grayscale erosion by replacing each pixel in the image with the lowest pixel intensity in that pixel's neighborhood $[5,6]$. Strut signals in the original IVUS images were eroded by the minimum filter, and the stentoriented 3D-IVUS was reconstructed from these minimum-filtered IVUS images (Fig. 1B). No fused strut images in the stent-oriented 3D-IVUS were seen, and struts and links could be well confirmed. It took about only $5 \mathrm{~min}$ from export of the IVUS image data to observation of the stent-oriented 3D-IVUS.

In another clinical case (Fig. 1C), Ultimaster Tansei was deployed from proximal to distal main vessel of a bifurcation lesion, and the guidewire was recrossed to the side-branch. The stent-enhanced 3D-OCT [6] of the jailed SBO is shown in Figure 1D. Using the stent-oriented 3D-IVUS, the jailing type and rewiring position on the jailed SBO could be confirmed as a link-connecting type and proximal rewiring (Fig. 1E) [1-3].

Intravascular ultrasound cannot detect struts unlike OCT/OFDI, and the stent-oriented IVUS simply makes it easier to confirm stents. Linearlink type stents such as Xience Sierra (Abbott Vascular, Santa Clara, CA, USA) may be easy to con-

Address for correspondence: Dr. Fumiaki Nakao, Department of Cardiology, Yamaguchi Grand Medical Center, 10077 Ohsaki, Hofu, Yamaguchi 747-8511, Japan, tel: +81-835-22-4411, fax: +81-835-38-2210, e-mail: nakao-ymghp@umin.ac.jp 

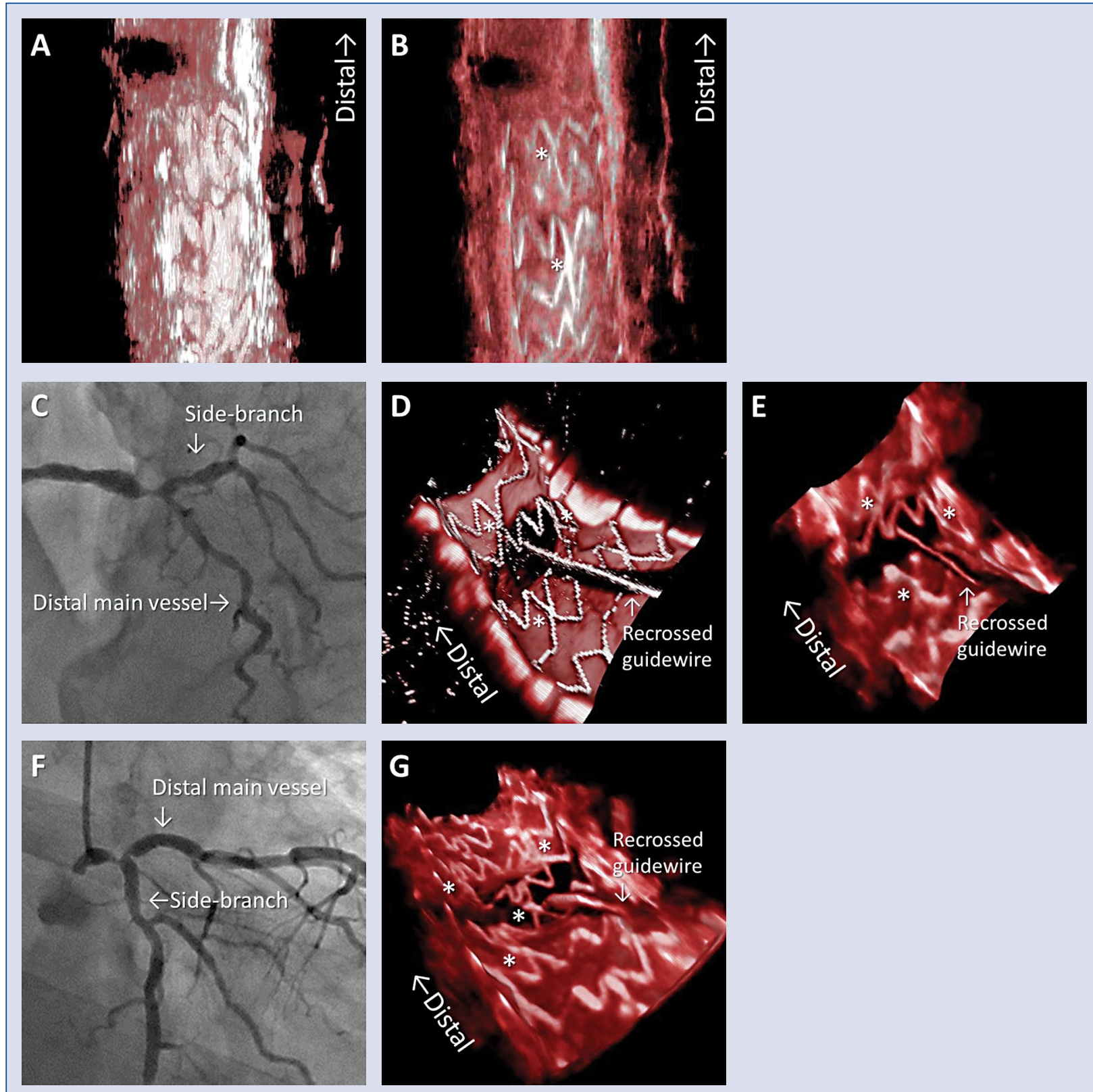

Figure 1. The stent-oriented three-dimensional (3D) intravascular ultrasound (IVUS). A. The longitudinal cut-away view of the 3D-IVUS reconstructed from the original IVUS images; B. The longitudinal cut-away view of the stent-oriented 3D-IVUS reconstructed from the minimum-filtered IVUS images. The baseline coronary angiogram (C), the longitudinal cut-away view of the stent-enhanced 3D optical coherence tomography (D) and the stent-oriented 3D-IVUS (E) of another clinical case (the crossover stent deployment on the bifurcation lesion with Ultimaster Tansei followed by guidewire recrossing). The jailing type and rewiring position can be confirmed by the stent-oriented 3D-IVUS as the link-connecting type and proximal rewiring. The baseline coronary angiogram (F) and the longitudinal cut-away view of the stent-oriented 3D-IVUS (G) of a clinical case with Xience Sierra (the crossover stent deployment on the bifurcation lesion followed by guidewire recrossing). Asterisks indicate stent links.

firm their links in a 3D-image reconstructed from sectional images because of longitudinally long links (Fig. 1F, G). Peak-to-valley type stents such as Ultimaster Tansei may be useful for the stentoriented 3D-IVUS even if they are the point-link type, because their adjacent struts, excluding those that are a link part, are apart from one another. In stent-oriented 3D-IVUS, flushing of blood cells by contrast medium is required to segment the vessel lumen, and flushing speed of contrast medium 
is the same as OCT/OFDI $(3.5-4.0 \mathrm{~mL} / \mathrm{s})$. Since only flushing of blood cells around the bifurcation is required, the amount of contrast medium for flushing in the stent-oriented 3D-IVUS is $5-10 \mathrm{~mL}$ and less than that in OCT/OFDI. Flushing of blood cells by low-molecular-weight dextran lactate may substitute for that by contrast medium [7], and the confirmation of the vascular lesion and the stent apposition in IVUS-guided PCI does not require flushing of blood cells basically. Most coronary interventional cardiologists are familiar with IVUS as an economical intravascular imaging devise. Adding this option (the 3D-reconstruction of the minimum-filtered IVUS images) to the IVUS console may result in good stent apposition on the SBO in IVUS-guided bifurcation PCI.

\section{Acknowledgements}

The author would like to thank Tatsuhiro Fujimura and Takayuki Okamura of the Division of Cardiology, Department of Medicine and Clinical Science of Yamaguchi University Graduate School of Medicine for their helpful advice.

Conflict of interest: Fumiaki Nakao received honoraria for technical consulting from TERUMO.

\section{References}

1. Okamura T, Onuma Y, Yamada J, et al. 3D optical coherence tomography: new insights into the process of optimal rewiring of side branches during bifurcational stenting. EuroIntervention. 2014; 10(8): 907-915, doi: 10.4244/EIJV10I8A157, indexed in Pubmed: 24531393.

2. Okamura T, Nagoshi R, Fujimura T, et al. Impact of guidewire recrossing point into stent jailed side branch for optimal kissing balloon dilatation: core lab 3D optical coherence tomography analysis. EuroIntervention. 2018; 13(15): e1785-e1793, doi: 10.4244/EIJ-D-17-00591, indexed in Pubmed: 29131806.

3. Nagoshi R, Okamura T, Murasato Y, et al. Feasibility and usefulness of three-dimensional optical coherence tomography guidance for optimal side branch treatment in coronary bifurcation stenting. Int J Cardiol. 2018; 250: 270-274, doi: 10.1016/j.ijcard.2017.09.197, indexed in Pubmed: 29030141.

4. KEN kun. AviUtl, available at. http://spring-fragrance.mints. ne.jp/aviutl/.

5. Rasband WS. ImageJ, U. S. National Institutes of Health, 1997-2018. http://imagej.nih.gov/ij/.

6. Nakao F, Ueda T, Nishimura S, et al. Novel and quick coronary image analysis by instant stent-accentuated three-dimensional optical coherence tomography system in catheterization laboratory. Cardiovasc Interv Ther. 2013; 28(3): 235-241, doi: 10.1007/ /s12928-013-0161-4, indexed in Pubmed: 23355032.

7. Ozaki Y, Kitabata H, Tsujioka H, et al. Comparison of contrast media and low-molecular-weight dextran for frequency-domain optical coherence tomography. Circ J. 2012; 76(4): 922-927, indexed in Pubmed: 22301848. 